\title{
Mechanical properties and carbon footprint of 3D-printable cement mortars with biochar additions
}

\author{
Devid Falliano ${ }^{1, *}$, Dario De Domenico ${ }^{2}$, Salvatore Quattrocchi $^{2}$, Paolo Cosenza $^{2}$, Giuseppe \\ Ricciardi $^{2}$, Luciana Restuccia ${ }^{1}$ and Giuseppe Andrea Ferro ${ }^{1}$ \\ ${ }^{1}$ Department of Structural, Geotechnical and Building Engineering, Politecnico di Torino, Corso Duca \\ degli Abruzzi, 24, Turin, 10129, Italy \\ ${ }^{2}$ Department of Engineering, University of Messina, Contrada Di Dio, 98166 Sant'Agata, Messina, \\ Italy
}

\begin{abstract}
This contribution focuses on the design and the characterization of innovative mix designs of high consistency mortars with biochar additions in different percentage with respect to the cement weight. Biochar is a by-product material that gives the cementitious mix a sustainable connotation from an environmental point of view. The mix designs presented here are characterized by a good dimensional stability in the fresh state, peculiarity that gives them the possibility to be extruded and so, to be used in automated construction processes. In addition to the mechanical properties (flexural and compressive strength), the assessment of the $\mathrm{CO}_{2}$ emission of representative mixes is presented. Different biochar content and maximum diameter of the aggregate are studied, obtaining interesting indications on these parameters to optimize mechanical properties. Finally, on the basis of the $\mathrm{CO}_{2}$ emission assessment, certain venues for future research work to minimize $\mathrm{CO}_{2}$ emissions are reported.
\end{abstract}

\section{Introduction}

Environmental sustainability in the construction field is an interesting research topic attracting increasing attention in recent years, considering the media impact on the climatic changes. Following this increasing interest regarding the environmental sustainability, different studies in the literature aimed at minimizing the so-called "environmental cost" of the most widely used material in the construction field, i.e. concrete. However, the attempt at optimizing raw materials or re-using waste products from the same or other industrial fields should be accompanied by acceptable performance requirements of the mixes, not only in terms of mechanical strengths, but also in terms of durability, especially for structural applications.

With regard to the optimization of raw materials, a possible strategy would be to use innovative production processes that make use of the mechatronics. The automation of the

\footnotetext{
* Corresponding author: dfalliano@unime.it
} 
construction process by means of the so-called "self-supporting" cementitious materials, i.e. materials that can withstand their self-weight in the fresh state without formworks [1], would allow a much wider freedom of design in terms of shapes. Additionally, it would also allow the use of particularly complex sections, by allocating the materials only where it is actually needed - which is often called "topology optimization" [2]. All these advantages would be useful for the design process, not only for saving material, but also to realize engineered and sophisticated structures.

Another possibility would be to modify the mix design of cementitious materials by using the traditional production processes, but replacing certain ingredients that are considered "energy intensive", expensive and non-renewable, with other ingredients from other industrial processes or recycled materials [3]. Alternatively, rather than using waste materials as replacement of traditional materials, another possibility would be to resort to special concretes, such as foamed concrete [4,5], wherein a part of the volume is filled with the most cost-effective ingredient in nature: air. In this case, the most difficult task is to use this promising material for structural applications, because unfortunately the presence of air voids, which reduces the volume mass, contributes to the worsening of the related mechanical performances. Hence, different strategies to improve the mechanical characteristics of the foamed concrete were developed: the addition of randomly distributed short fibers in the mix [6], bi-directional grid reinforcement [7], or additions of ingredients having pozzolanic activity [8].

Based on the above remarks, the development of innovative and eco-friendly materials is a hot research topic. Along this research line, in this contribution, certain cementitious mixes are presented that combine environmental sustainability, aim to optimize the production process, with the introduction of byproducts in the mix design. In particular, a series of mortars are presented in this paper that are characterized by the use of biochar and by a high consistency that gives rise to an optimal dimensional stability in the fresh state, which enables its potential use in automated production processes. The influence of different biochar contents in the percentage of weight with respect to the cement and the influence of the aggregate to cement ratio on the compressive strength as well as on the flexural strength of the mix are scrutinized. Finally, the $\mathrm{CO}_{2}$ emission corresponding to the considered mix designs is also assessed and discussed.

\section{Materials and methods}

The mortars are prepared with Portland CEM I 52,5 R in accordance with UNI EN 197-1. With regard to the aggregate, volcanic sand having the maximum diameter of $1 \mathrm{~mm}$ is adopted. The choice of this maximum diameter is related to the need of avoiding possible obstructions of flow during the pouring of the cement materials from the extrusion nozzles during a $3 \mathrm{D}$ printing process. Indeed, the aim is to develop a mix capable of self-bearing its weight in the fresh state, so as to be usefully employed in automated production processes. Typical dimensions of extrusion nozzles of 3D printers are usually in the range of 10-60 $\mathrm{mm}$. In particular, three different aggregate/cement ratios are adopted: $0.72,1.28$ e 2.78 . In Figure 1, the grain size distribution curve of the employed aggregate is illustrated.

As said above, the mixes are prepared with the addition of biochar, so as to give the prepared material a green connotation. Moreover, in the relevant literature on both traditional cements [9] and special concretes, in particular foamed concretes [10], it has been found that the biochar improves certain characteristics of the mix, more specifically its fracture energy. The percentage of biochar varies from 5\% to $22.7 \%$ of the cement weight. In order to keep the water content as small as possible, a superplasticizer is added in some mixes, namely the Master Ease 7000 produced by Basf, which is an additive based on polycarboxylates ethers. In this research, the use of this superplasticizer does not primarily 
aim to obtain a low-viscosity concrete because the objective is to obtain 3D-printable mortars. Instead, the employed superplasticizer aims to reduce the water/cement ratio in presence of biochar in order to obtain high resistances. In general, the superplasticizer percentage varies from 0 to $12 \%$ of the cement weight. Tap water varying from $27 \%$ to $51 \%$ of the cement weight is used.

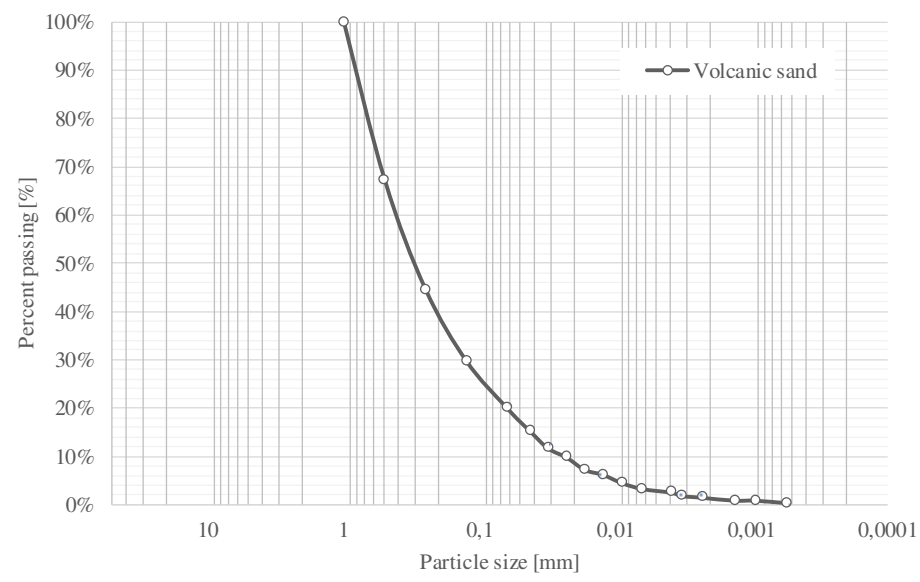

Fig. 1. Grain size distribution curve of the employed volcanic sand.

In order to report this mix proportions in the designation of the specimen, the nomenclature adopted is $\mathrm{M} \_\mathrm{BCi} \mathrm{V}_{\mathrm{V}} \_\mathrm{SPk}$ _Wy, where $\mathrm{M} \_\mathrm{BC}$ stands for mortar with the biochar addition, $\mathrm{i}$ represents the biochar content in the percentage of cement weight, $\mathrm{V}$ represents the volcanic sand used, and $\mathrm{j}$ is the sand-to-cement ratio, SP represents the superplasticizer and $\mathrm{k}$ is its percentage (of the cement weight), finally $\mathrm{W}$ represents water and $y$ is the related percentage of water in relationship to the cement weight. The research program includes the following range of parameters: aggregate/cement ratio equal to 70 , 127 and $280 \%$; biochar/cement ratio equal to $5,9,11$ and $23 \%$; water/cement ratio equal to $28,37,39$ and 51\%; superplasticizer/cement ratio equal to $0,5,6$, and $10 \%$.

Table 1. Mix proportions of the mortar samples considered in the experimental campaign.

\begin{tabular}{|c|c|c|c|c|}
\hline Nomenclature & $\begin{array}{c}\text { biochar/cement } \\
\text { ratio }(\%)\end{array}$ & $\begin{array}{c}\text { aggregate/cement } \\
\text { ratio }(\%)\end{array}$ & $\begin{array}{c}\text { superpl./cement } \\
\text { ratio }(\%)\end{array}$ & $\begin{array}{c}\text { water/cement } \\
\text { ratio }(\%)\end{array}$ \\
\hline M_BC 11 V $280 \_S P_{10} W_{37}$ & 11 & 280 & 10 & 37 \\
\hline 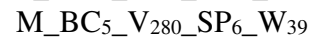 & 5 & 280 & 6 & 39 \\
\hline M_BC $23 \_V_{280} S_{S_{0}} W_{51}$ & 23 & 280 & 0 & 51 \\
\hline M_BC9_-V70_SP 5 -W 28 & 9 & 70 & 5 & 28 \\
\hline $\mathrm{M} \_\mathrm{BC} \mathrm{C}_{9} \mathrm{~V}_{127} \mathrm{SP}_{5} \mathrm{~W}_{28}$ & 9 & 127 & 5 & 28 \\
\hline
\end{tabular}

The specimens are prepared in accordance with UNI EN 196-1 (dimensions $40 \mathrm{x} 40 \mathrm{x}$ $160 \mathrm{~mm}$ ) and after 28 days of standard laboratory curing, they are tested with a CONTROLS test frame model 65-L1301/FR in two steps: the first step is the flexural test, then the two halves of the broken prism are tested in compression. Both tests are carried out in the force-controlled mode, with a load rate of $50 \mathrm{~N} / \mathrm{s}$ and $2500 \mathrm{~N} / \mathrm{s}$ for the flexural test and for the compressive test, respectively.

Besides the mechanical strengths, the $\mathrm{CO}_{2}$ emission of the prepared mixes is also evaluated. The evaluation of $\mathrm{CO}_{2}$ emissions has been performed based on the amount of $\mathrm{CO}_{2}$ emissions for each component of the mix design, which was established according to previous literature studies. Then, the estimation of the overall $\mathrm{CO}_{2}$ emission is calculated as the sum of each component. It should be noted that the present study is limited to the 
assessment of the $\mathrm{CO}_{2}$ emission related to the production phase. Therefore, this study does not include the $\mathrm{CO}_{2}$ emissions caused by the casting in the construction site, nor the $\mathrm{CO}_{2}$ emissions related to the transportation from the manufacturing place to the concrete plant, nor the lifecycle data (e.g. recovering part of $\mathrm{CO}_{2}$ emissions through the carbonation of concrete [11].

It is worth noting that the cement is the main material that causes the high production of $\mathrm{CO}_{2}$. The cement-related emissions vary quite largely depending on the country of production, because each country has its own mix of resources to produce energy and different technologies to produce the clinker [12]. In this study, the assumption of producing the cement in Europe was decided upon. In the $\mathrm{CO}_{2}$ evaluation of this work, the contribution of biochar is neglected. Future work is ongoing to assess the positive contribution of biochar with regard to the $\mathrm{CO}_{2}$ emissions.

\section{Mechanical properties}

In this section, results in terms of flexural strength and compressive strength are reported and discussed for the considered mortars with biochar, having high consistency as shown in Figure 2. In particular, the influence of the biochar content (in relation to the cement weight) and of the aggregate-to-cement ratio on the mechanical properties are discussed. The research plan and the investigated specimens cover different parameters in terms of water/cement ratio, aggregate/cement ratio and biochar/cement ratio. The aim is to highlight the significant influences that the variations of these parameters, despite being limited to within a range that does not alter the high-consistency for possible 3D-printing applications of the mortars, have on the mechanical strength.

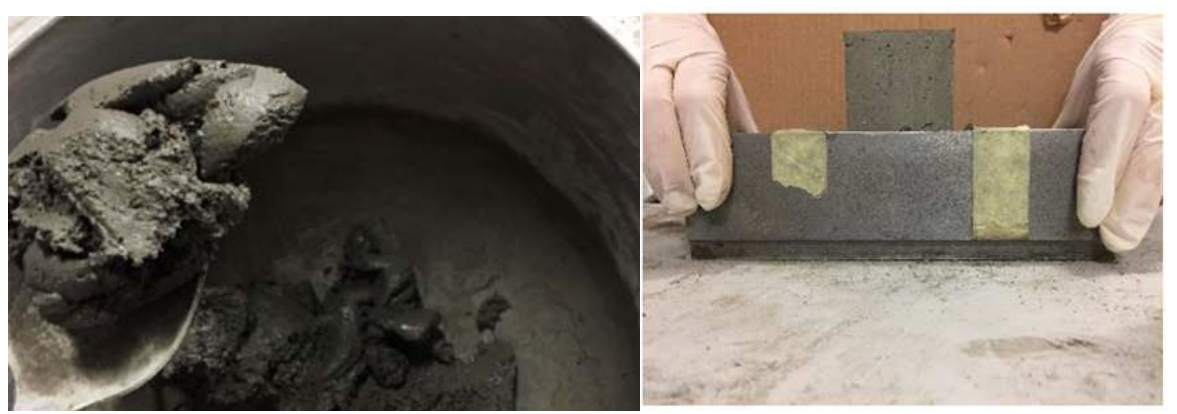

Fig. 2. Representative photograph of the high consistency of the mortars with biochar additions (left) and extrusion test to assess the suitability for 3D printing applications (right).

The consistency of the analyzed mortars is evaluated through an extrusion test aimed at assessing the dimensional stability of the pastes in the fresh state and the resulting suitability for 3D printing applications, as described in Falliano et al. [13] and sketched in the right part of Figure 2.

In particular, in the comparative histogram reported in Figure 3, the effect of the biochar content is investigated for the mixes having the highest aggregate-to-cement ratio among those analyzed in the present study. It is noted that a reduction of the biochar content from $11 \%$ to $5 \%$ of the cement weight leads to an increase of around $17 \%$ and $15 \%$ of the compressive and flexural strength values, respectively. The small differences in terms of water-to-cement ratios ( $37 \%$ and $39 \%$, respectively) are compensated by a modification of the amount of superplasticizer (10\% and 6\%, respectively). 


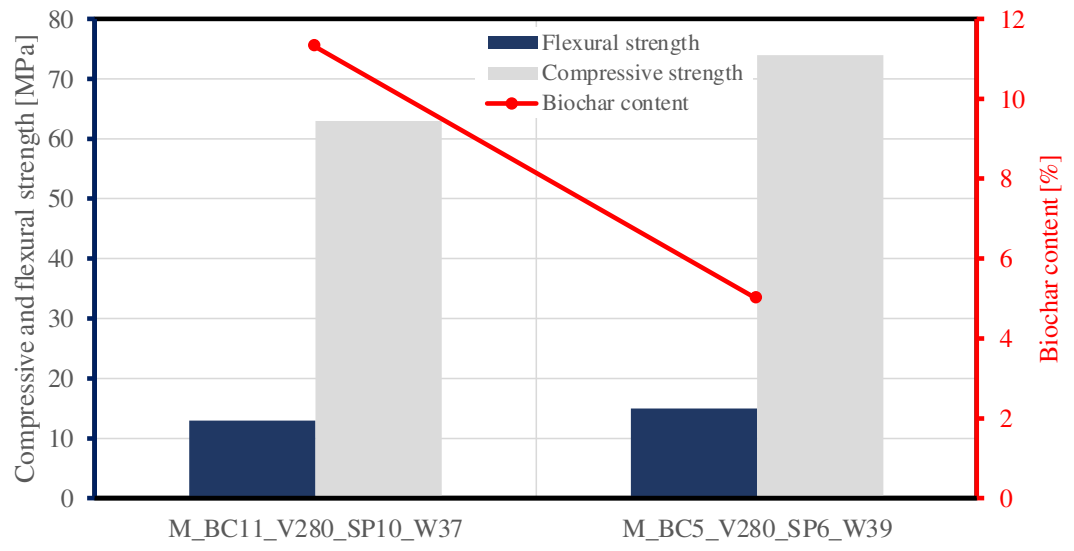

Fig. 3. Comparative histogram expressing the influence of the biochar quantity on the mechanical strengths of cementitious mortars with the same aggregate content (aggregate/cement $=280 \%$ ) and similar water/cement ratios (37 and 39\%).

In Figure 4, a very interesting comparison among specimens prepared with the same sand-to-cement ratio but different biochar-to-cement and water-to-cement ratios is shown. It can be noticed that the further increase of the biochar content from $11 \%$ to $23 \%$ with respect to the cement weight does not lead to a dramatic decrease of the strengths, which turn out to be comparable in the two analysed cases. A possible justification can be obtained by analysing the other differences in terms of the mix design of the mixes, more specifically the different water-to-cement ratio that is $37 \%$ in the first case (with the necessary addition of the superplasticizer), and $51 \%$ in the second case (without the superplasticizer). Despite the high water-to-cement ratio, the second mix is also characterized by a high consistency in the fresh state due to the water absorption property of biochar. The high strength achieved, comparable to those obtained with a much lower water-to-cement ratio, could be due to the water issue of the biochar during the first stages of the cement curing process, thus improving the hydration and acting as an internal curing agent. This peculiar effect of the biochar, here only qualitatively analyzed and discussed, will be more studied in detail in prospective research works.

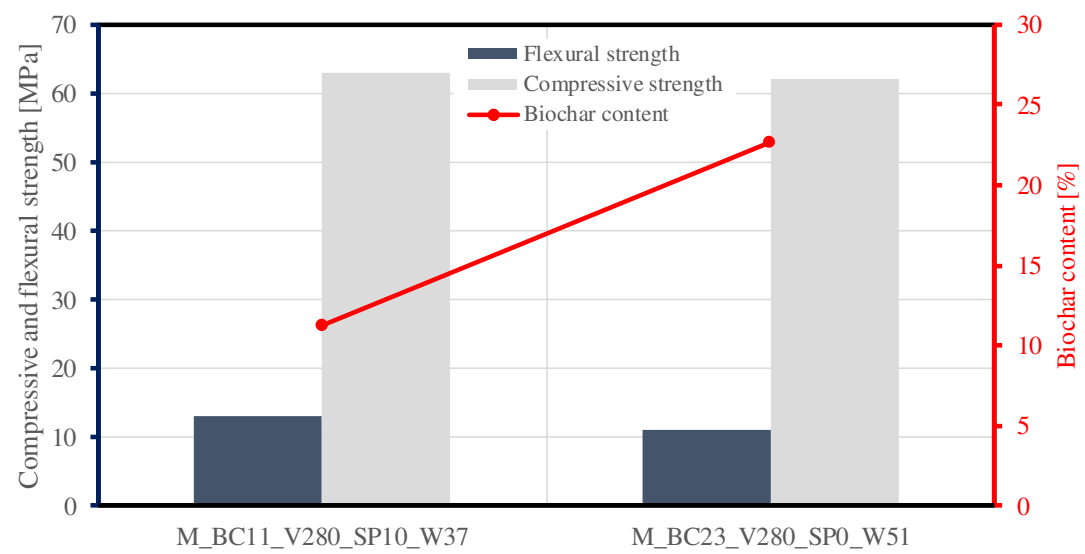

Fig. 4. Comparative histogram expressing the influence of the water and biochar content on the mechanical strengths of cement mortars with the same aggregate content (aggregate/cement $=280 \%$ ). 


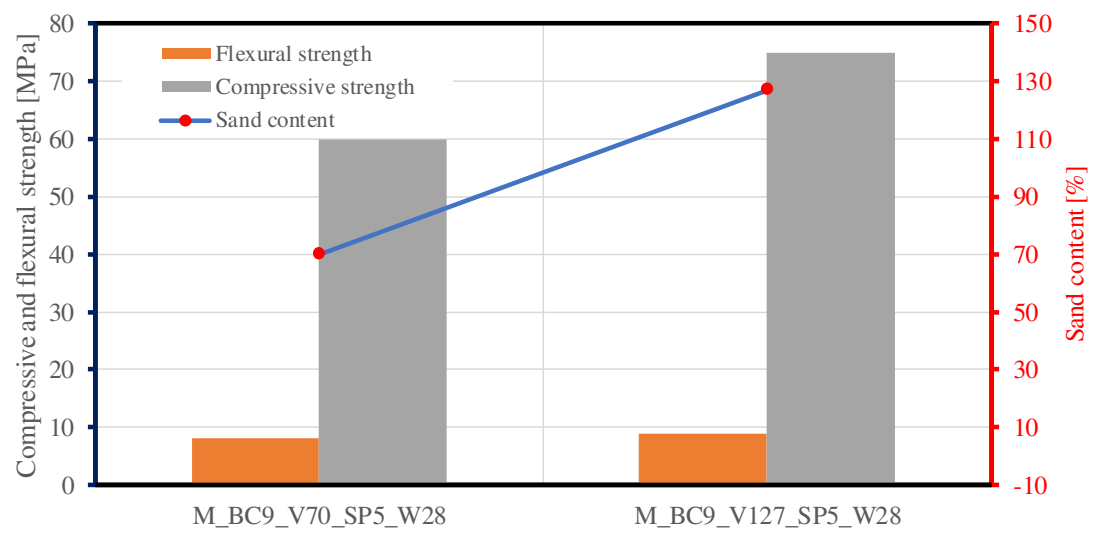

Fig. 5. Comparative histogram expressing the influence of the aggregate content on the mechanical strengths of cementitious mortars with the same biochar content (biochar/cement $=9 \%$ ) and the same water/cement ratio $(28 \%)$.

In Figure 5, the influence of the sand-to-cement ratio on the mechanical strength for equal conditions in terms of the biochar content, superplasticizer, cement and water-tocement ratios is illustrated. It can be seen that the increase of the sand content from $70 \%$ to $127 \%$ of the cement weight produces an increase of the mechanical strengths of $25 \%$ and of $12 \%$ for the compressive and flexural strength (due to the higher aggregate interlock effect), respectively.

\section{$4 \mathrm{CO}_{2}$ assessment}

After the analysis of the mechanical tests, the evaluation of $\mathrm{CO} 2$ emission was examined. It is important to highlight that the contribution of biochar to the $\mathrm{CO}_{2}$ emission is neglected in the calculations, because it is a waste product from industrial processes. Further investigation is ongoing to evaluate the positive contribution related to the use of biochar in the cement mixes, which will be discussed in subsequent research papers. However, it is worth noting that neglecting biochar is a conservative evaluation, because when biochar is taken into account, the $\mathrm{CO}_{2}$ emission is expected to be lower than that presented in this paper. To this aim, certain representative mixes were selected to highlight the influence of different biochar/cement ratios and aggregate/cement ratios on the carbon footprint. The choice of these representative specimens is aimed to include different biochar contents (from low to high contents) and different aggregate/cement ratios. In particular, in Figure 6, the evaluation of the $\mathrm{CO}_{2}$ emission is reported for three mixes having biochar contents of $5 \%, 9 \%$ and $23 \%$ with respect to the cement weight, and sand-to-cement ratios of $127 \%$ and $280 \%$. As said above, the main culprit concerning the $\mathrm{CO}_{2}$ emission is the cement, which clearly explains the results shown in Figure 6. For comparison purposes, the carbon footprint of a standard mortar without biochar additions and without the superplasticizer (with the same dimensional stability and high resistance) is also reported in Figure 6. This mortar is characterized by aggregated/cement ratio approximately equal to the highest one (280\%) employed for the mortars with biochar. It is worth noting that the carbon footprints of individual mortar components assumed in this calculations are: 0.86 $\mathrm{kgCO}_{2} / \mathrm{kg}$ of material for cement; $0.0018 \mathrm{kgCO}_{2} / \mathrm{kg}$ of material for sand; $0.39 \mathrm{kgCO}_{2} / \mathrm{kg}$ of material for superplasticizer; $0.0001 \mathrm{kgCO}_{2} / \mathrm{kg}$ of material for water. These values are assumed based on technical specifications from manufacturers' websites and other relevant 
literature studies [14]. By analysing these figures, it is clear that the mix design characterized by the highest biochar content and the highest sand-to-cement ratio is associated with lower $\mathrm{CO}_{2}$ emission. The highest $\mathrm{CO}_{2}$ emission is obtained for the mix with the biochar content of $9 \%$ of the cement weight, which is ascribed to the lower sand-tocement ratio used in this mix and, consequently, to a higher amount of cement per cubic meter of material. It can be noted that, by properly adjusting different proportions of the various ingredients of the mix design, it is possible to obtain the reduction of the $\mathrm{CO}_{2}$ emission of around $43 \%$ with compressive strengths that are still pertinent to highresistance concretes (i.e. higher than $50 \mathrm{MPa}$ ). Finally, it should also be noted that, generally, the addition of biochar produces a reduction of the $\mathrm{CO}_{2}$ emission compared to a standard mortar for equal aggregate/cement ratio of $280 \%$. Obviously, the samples with low sand-to-cement ratio (and, hence, a higher cement content) are associated with higher carbon footprint.

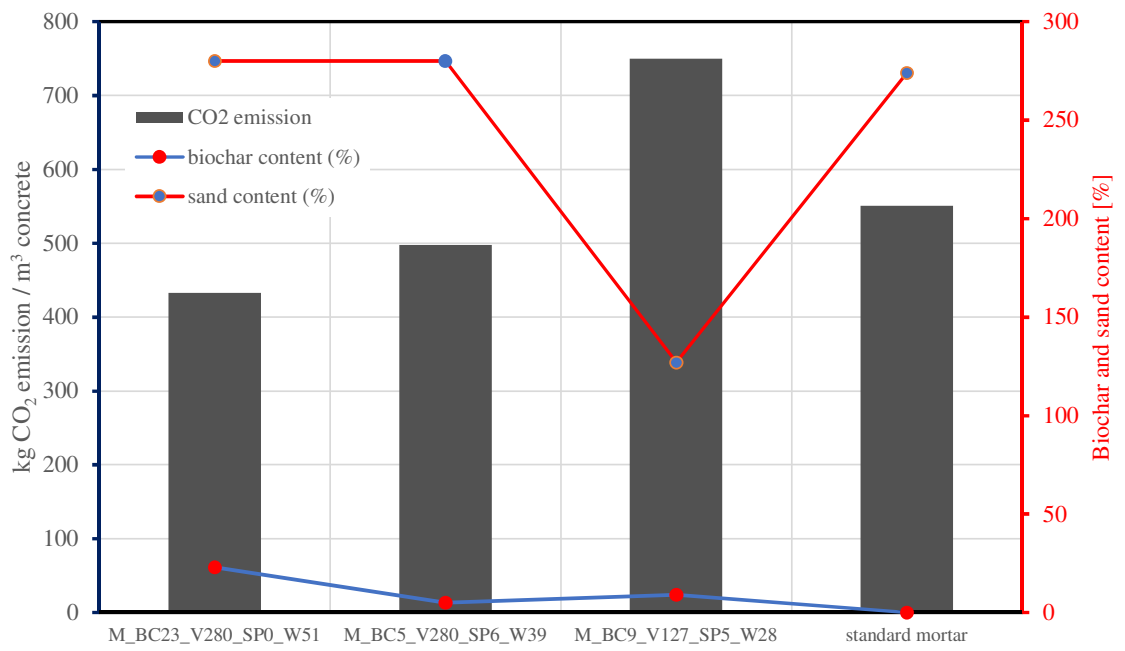

Fig. 6. $\mathrm{CO}_{2}$ emission evaluation for certain representative mix designs.

\section{Conclusions}

This contribution has presented the results of an experimental investigation on the mechanical strength of mortars containing biochar and characterized by high consistency in the fresh state. The goal is to obtain cementitious mixes that are able to reduce the environmental impact with a twofold strategy: 1) resorting to waste materials, in this case biochar; 2) using innovative technologies for production, like digital fabrication, which is feasible owing to excellent dimensional stability in the green state. To reach this goal, a key role is played by the addition of biochar that appropriately modifies the rheological properties of the mixes in the fresh state. The results indicated that it is possible to obtain high-resistance mixes (with compressive strengths higher than $60 \mathrm{MPa}$, with a peak value of $75 \mathrm{MPa}$, and flexural strengths higher than $8 \mathrm{MPa}$, with a peak value of $15 \mathrm{MPa}$ ) with the use of modest quantities of biochar as well. Some results revealed a possible effect of internal curing of biochar. Following these results, future studies will aim to further investigate this very interesting property. Finally, the contribution of the different mixes proposed in terms of $\mathrm{CO}_{2}$ emission was also assessed with simple calculation tools. Considering the significant contribution of the cement in such an evaluation, the mixes 
characterized by the highest biochar-to-cement ratio and sand-to-cement ratios turned out to be those associated with the lower $\mathrm{CO}_{2}$ emissions. By properly optimizing the mix design, it was demonstrated that it is possible to reduce the $\mathrm{CO}_{2}$ emission even by $43 \%$ while maintaining mechanical strength to very high values, typical of high-resistance mortars.

\section{Acknowledgements}

The authors wish to thank the company Colacem S.p.A. for providing the cement and the company BASF for providing the superplasticizer. In particular, the authors warmly thank Dr. Sandro Moro for the useful suggestions.

\section{References}

1. D. Falliano, E. Gugliandolo, D. De Domenico, G. Ricciardi, RILEM International Conference on Concrete and Digital Fabrication - Digital Concrete 2018, Wangler T., Flatt R. (eds.), 19, 277-286, DOI: 10.1007/978-3-319-99519-9_26 (2018).

2. P. Martens, M. Mathot, F. Bos, J. Coenders, High Tech Concrete: Where Technology and Engineering Meet, 301-309 (2018).

3. J.A. Bogas, J. de Brito, J.M. Figueiredo, J. Clean. Prod. 89, 187-195 (2015).

4. D. Falliano, D. De Domenico, G. Ricciardi, E. Gugliandolo, Constr.Build. Mater. 165, 735-749 (2018).

5. D. Falliano, D. De Domenico, A. Sciarrone, G. Ricciardi, L. Restuccia, J.M.C. Tulliani, E. Gugliandolo, Theor. Appl. Fract. Mech. 103, 102297 (2019).

6. D. Falliano, D. De Domenico, G. Ricciardi, E. Gugliandolo, Constr.Build. Mater. 198, 479-493 (2019).

7. D. Falliano, D. De Domenico, G. Ricciardi, E. Gugliandolo, Compos. Struct. 209, 4559 (2019).

8. M.R. Jones, A. McCarthy, Mag. Concr. Res. 57, 1, 21-31 (2005).

9. L. Restuccia, G.A. Ferro, Constr. Build. Mater. 126, 1034-1043 (2016).

10. D. Falliano, D. De Domenico, A. Sciarrone, G. Ricciardi, L. Restuccia, G. Ferro, J.M. Tulliani, E. Gugliandolo, Frattura ed Integrità Strutturale, 14, 51, 189-198 (2020).

11. R. Leese, D. Casey, Report of Mineral Products Association (MPA), Fact Sheet 18, (2012).

12. R. Kajaste, M. Hurme, J. Clean. Prod. 112, 4041-4052 (2016).

13. D. Falliano, D. De Domenico, G. Ricciardi, E. Gugliandolo, Constr.Build.Mater. 254, 119271 (2020).

14. R. Leese, D. Casey, Embodied CO2e of UK cement, additions and cementitious material. Report of Mineral Products Association (MPA), 2012, Fact Sheet 18. 